\title{
Boundary layer flow over a moving surface in a nanofluid with suction or injection
}

\begin{abstract}
An analysis is performed to study the heat transfer characteristics of steady two-dimensional boundary layer flow past a moving permeable flat plate in a nanofluid. The effects of uniform suction and injection on the flow field and heat transfer characteristics are numerically studied by using an implicit finite difference method. It is found that dual solutions exist when the plate and the free stream move in the opposite directions. The results indicate that suction delays the boundary layer separation, while injection accelerates it.
\end{abstract}

Keyword: Nanofluid; Moving plate; Boundary layer; Suction/injection; Dual solutions 